\title{
EDITORIAL
}

\section{Statistical significance in genetic association studies}

\author{
Hui-Qi Qu PhD ${ }^{1}$ \\ Matthew Tien' \\ Constantin Polychronakos MD $^{3}$
}

${ }^{1}$ University of Texas Health Science Center Houston,
School of Public Health, Brownsville, Texas, USA;
${ }^{2}$ University of Texas at Austin, School of Biological Sci-
ences, Austin, Texas, USA;
${ }^{3}$ The McGill University Health Center (Montreal Chil-
dren's Hospital), Montréal, Québec, Canada

Clin Invest Med 2010; 33 (5): E266-E270.
Clinical \& Investigative Medicine (CIM) is receiving an increasing number of reports of candidate-based association studies. The track record of such studies in the past has been poor: numerous genetic associations reported from candidate gene studies have not been replicated in later studies. ${ }^{1}$ The rise of the genomewide association study (GWAS) is changing this situation. A well-designed GWAS may identify a number of candidate loci without bias by screening the whole human genome. Validating and fine-mapping the candidate loci from GWAS are required to clarify the genetic mechanisms. Thus, a candidate-based association study has become a well-directed effort, instead of searching for a needle in a haystack. In the postGWAS era, exponential growth of candidate-based genetic association studies is expected. A pressing issue accompanying this new trend is the assessment of the validity of an association study. In this editorial, we illustrate the major cause of false positive association from random sampling bias by an empirical example, and emphasize the application of the probability theory in assessing the validity of a genetic association study.

For the majority of human common diseases, the etiology is not well understood. This lack of knowledge has impeded the development of effective strategies to prevent or cure the diseases. The effort to understand the molecular mechanisms of common diseases has been complicated by the fact that a single disease usually involves multiple etiological pathways, mediated by many of the approximately 20,000 to 25,000 protein-coding genes encoded in the human genome. ${ }^{2}$ With the completion of the Human Genome Project in 2003, investigation of human genetics has become an indispensable approach to understand the molecular basis of common diseases, and has penetrated every branch of biomedical science. An important aspect of human genetics is the highly polymorphic nature of the human genome. There are $>11$ million common DNA variants with frequencies $>1 \%$, i.e. DNA polymorphisms (the NCBI dbSNP database, http://www.ncbi.nlm.nih.gov/projects/SNP; the 1000 genome project, http://www.1000genomes.org). Except for identical (monozygotic) twins, no two individual's genomes are identical, although the difference between any two persons across the world is less than $0.1 \%$ of the whole genome. Because of the high incidence of DNA polymorphisms, different individuals may have different susceptibilities to a common disease. ${ }^{3}$ The diversity of genetic susceptibility to common diseases in the human population enables researchers to understand the molecular mechanisms of common diseases by the method of genetic association study, which examines the coexistence of genetic markers for a disease. 
In the analysis of genetic association studies, a parameter of statistical significance, a $p$-value, is used to assess the certainty of an association. A $p$-value provides the probability that a given result from a test is due to chance. A common cutoff for each test, $\alpha=0.05$, reflects a $95 \%$ likelihood that the result is not a coincidence; however, a genetic association study usually tests multiple genetic markers, therefore false positives will accumulate. If each test has $95 \%$ certainty, the liklihood that $\mathrm{n}$ tests will be true will lowered to $(95 \%)^{\mathrm{n}}$. A GWAS study implements sophisticated genomic technologies and involves an extraordinary number of hypotheses and statistical tests, thereby providing clear examples of the accumulated errors from multiple tests. To demonstrate the effect of accumulated errors empirically, we performed an experiment in type 2 diabetes (T2D) using a GWAS dataset (the Gene Environment Association Studies initiative, GENEVA, http://www.genevastudy.org), involving 3,148 patients and 2,745 controls. ${ }^{4}$ From the 871,166 autosome single-nucleotide polymorphisms (SNP) genotyped in this study, we removed all the SNPs with possible (statistically significant) disease association in this cohort (56,103 SNPs with $P \leq 0.05)$. From the remaining 815,063 SNPs, we kept only 278,869 SNPs with low linkage disequilibrium (LD), by eliminating all the other SNPs with $\mathrm{r}^{2} \geq 0.5$ with these SNPs, to assure that genotyping the retained 278,869 SNPs represented independent tests. Consequently, we performed a resampling experiment, i.e. 500 cases and 500 controls were randomly sampled from the GENEVA T2D cohort, and T2D associations were tested in this resampling cohort. We then repeated the resampling experiment 34 times. A large number of SNPs showed $\mathrm{P} \leq 0.05$ in each resampling cohort (Fig.1, $\bar{x} \pm s=11,641 \pm 1,059)$. As we had removed all the SNPs with $P \leq 0.05$ in the original cohort, we can assume that all these $P \leq 0.05$ in the resampling cohort are false positives due to random sampling bias. The average false positive rate is $4.17 \pm 0.38 \%$. Although we eliminated all the other SNPs with $r^{2} \geq 0.5$ with these SNPs, the remnant LD among SNPs explains the lower false positive rate from the smaller number of independent hypotheses. The false positives from random sampling bias also explain the transient positives that are commonly seen in low-throughput genetic association studies, and disappear with the genotyping of more DNA samples.

To address the accumulated errors from multiple tests, a valid statistical analysis requires a corrected significance cutoff. Two methods are commonly used to determine the corrected cutoff; i.e., the Bonferroni correction and the false discovery rate (FDR) correction. The Bonferroni correction is a stringent method that adjusts $p$-values by multiplying each $p$-value by the total number of tests. ${ }^{5}$ Only the adjusted p-values $\leq 0.05$ are taken as statistically significant. The False discovery rate (FDR) correction was introduced by Benjamini and Hochberg. ${ }^{6}$ The FDR method first ranks all $\mathrm{p}$-values from the smallest to the largest, and then adjusts each p-value accordingly:

$$
\text { FDR corrected } p=\frac{\text { Number of tests }}{p \text { Rank }} \times p
$$

FDR corrections are less stringent and tolerate more false positives; i.e., the FDR correction of FDR $=0.05$ allows $5 \%$ of reported positives are false positives, while the Bonferroni correction of $\alpha=0.05$ requires the whole family of positives to be true positives with the certainty of $95 \%$. For the smallest p-value in one study, the Bonferroni-corrected p-value is equal to the FDR-corrected p-value.

In our resampling experiment, both the Bonferroni correction and the FDR correction adequately addressed the issue of false positive values. No p-value from the random sampling bias has cleared either correction. The median of the minimum $\mathrm{p}$-value of each of the 34 resamplings is $8.75 \times 10^{-6}$, while the smallest p-value of the 34 resamplings is $3.89 \times 10^{-7}$. In our experiment, to obtain at least one p-value with significance using either the Bonferroni correction or FDR correction, the smallest $\mathrm{p}$-value should be less than $\alpha=0.05 / 278,869=1.79 \times 10^{-7}$. 


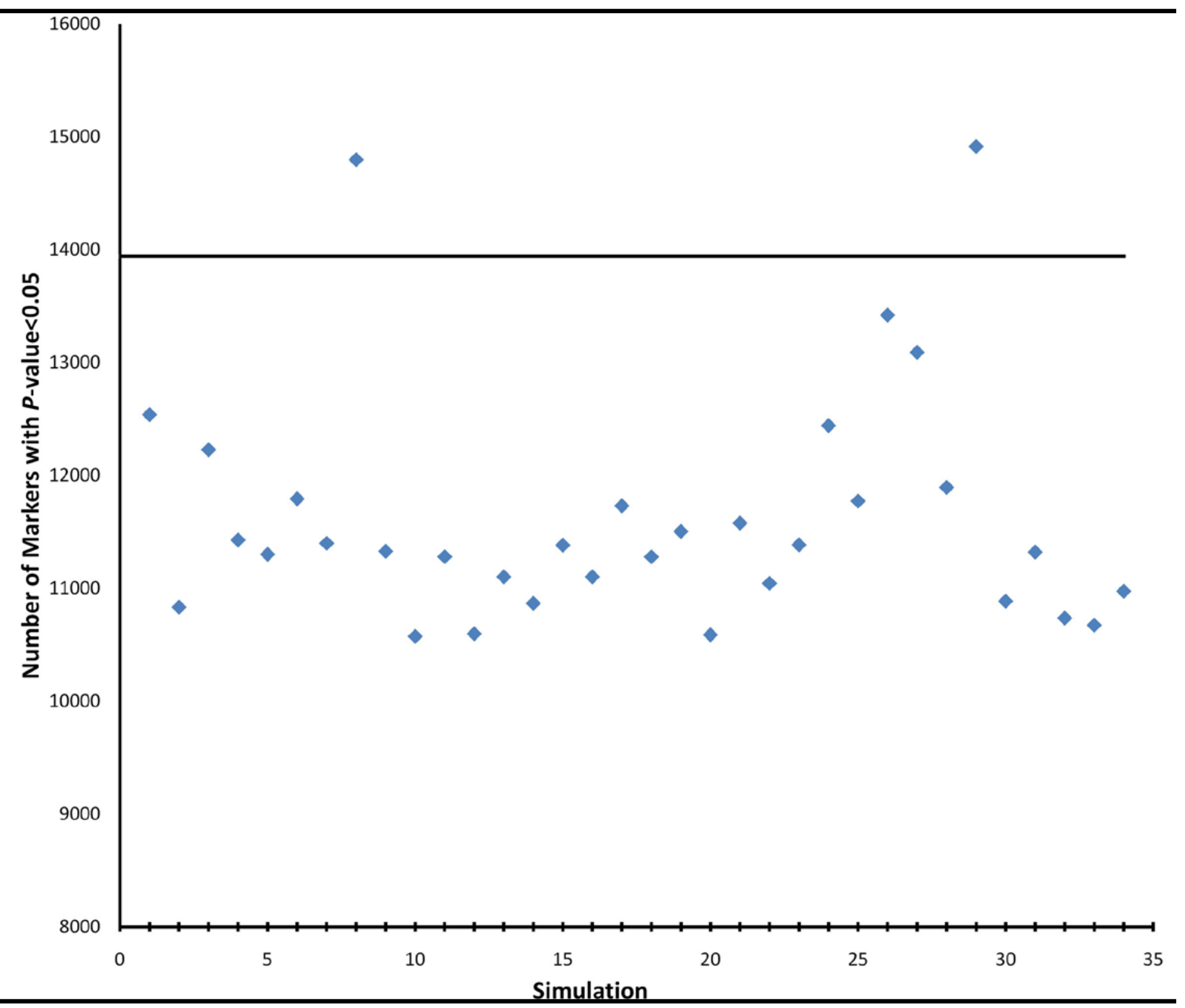

FIGURE 1. Number of false positive associations in the resampling experiment. X-axis represents 34 resamplings; Y-axis represents the number of tests with $P \leq 0.05$. The horizontal line corresponds to 13,943 false positives (i.e., $5 \%$ of 278,869 independent hypotheses by chance alone). The average false positive rate of $4.17 \%$ suggests that the 278,869 SNPs across the human genome are not completely independent because of linkage disequilibrium ( 232,577 independent hypotheses).

Statistical analysis provides the most critical criterion to assess the validity of the results of a biomedical research. GWAS studies are the perfect examples of the rigorous usage of probability theory to claim the significance of the results. Multiple testing corrections are indispensable for identifying true positives and sifting out false positives. The large number of false positives in GWAS studies highlights the importance of duly adjusting for multiple tests in the smaller scale and candidate-based genetic association studies. Because of the availability of inexpensive and highthroughput genotyping technology, the cost of DNA genotyping has been much lower than the cost of sample recruitment. A candidate-based genetic asso- 
ciation study usually tests multiple loci and markers. Yet, most association studies on candidate gene currently published (including those submitted to CIM) make no mention of the multiple genes that were tested and found not to be significant, only presenting those with $\mathrm{p}<0.05$. Although it is conceivable that hundreds or thousands of DNA samples were really collected to test a single candidate gene and then destroyed, this is a totally implausible scenario that certainly does not apply in the majority of these reports.

In summary, to ensure the validity of a genetic association article, a study with a definite number of hypotheses (e.g., a GWAS study) should be supported by the statistical significance after correction for multiple testing comparisons, and the total number of hypotheses used for statistical correction should be justified. Attention is needed for the data interpretation of such studies: if a GWAS study reports a number of positive loci (and the related molecular pathways), statistical significance after the less stringent FDR correction would be sufficient; however, for researchers to draw conclusions on a specific genetic locus, statistical significance after Bonferroni correction is required. For a candidate-based study in which the total number of hypotheses cannot be justified indubitably, the association needs to be validated. An independent cohort that has a definite number of hypotheses to be validated can usually offer sufficient statistical evidence. Published GWAS studies on the same phenotype can be a excellent resource for validation. To date, a large number of GWAS data are publicly available in two major GWAS databases; i.e., the NCBI database of Genotypes and Phenotypes (dbGaP, http://www.ncbi. nlm.nih.gov/gap), and the European GenomePhenome Archive (EGA, http://www.ebi.ac.uk/ega). The replication of an association in a public GWAS dataset may be limited by the coverage of the GWAS SNP genotyping arrays. In this case, in silico replication of the association can be done in the GWAS cohort. Depending on the ethnic background of the GWAS subjects, one of the 11 populations genotyped in the HapMap study (phase 2 and phase 3, Feb 09
Release, http://www.hapmap.org) can be used as the reference population. Interesting DNA polymorphisms need to be genotyped in the reference population if this population has not been genotyped by the HapMap project. DNA samples of different populations can be acquired from the Human Population Collections of the Coriell Cell Repositories (http://ccr.coriell. org). Consequently, the genotypes and association in the GWAS study can be input using the hidden Markov Chain based algorithm; e.g., the MACH software (http://www.sph.umich.edu/csg/abecasis/MaCH/index. html).

Limited by the low-throughput genotyping technology, previous association studies had to focus on genes with candidate functions, which had little value in helping us to understand the unknown aspects of a disease. Because a GWAS is hypothesis-free, it has turned out to be enormously helpful in understanding the unknown aspects of many common human diseases by allowing the unbiased examination of hundreds of thousands of genetic markers across human genome for disease associations. ${ }^{3}$ On the other hand, a GWAS still has limited statistical power and limited coverage of human genomics. A GWAS may suggest many interesting loci; however, to draw conclusions concerning a novel molecular mechanism in a disease, the association of the candidate loci needs to be validated and fine-mapped. The method of the genetic association study is becoming more efficient in helping us understand human diseases. The probability theory is a critical fundament of modern biomedical research, which has been strictly applied in GWAS studies, and should not be neglected in candidate gene studies.

\section{References}

1. Kavvoura, F.K. and J.P. Ioannidis, Methods for meta-analysis in genetic association studies: a review of their potential and pitfalls. Hum Genet, 2008. 123(1): p. 1-14. 
2. Stein, L.D., Human genome: End of the beginning. Nature, 2004. 431(7011): p. 915-916.

3. Manolio, T.A., L.D. Brooks, and F.S. Collins, $A$ HapMap harvest of insights into the genetics of common disease. J Clin Invest, 2008. 118(5): p. 1590-605.

4. Cornelis, M.C., et al., Joint effects of common genetic variants on the risk for type 2 diabetes in U.S. men and women of European ancestry. Ann Intern Med, 2009. 150(8): p. 541-50.

5. Miller, R.G.J., ed. Simultaneous Statistical Inference Springer Series in Statistics. 1981, Springer. 6-8.

6. Benjamini, Y. and Y. Hochberg, Controlling the false discovery rate: a practical and powerful approach to multiple testing. JOURNAL- ROYAL STATISTICAL SOCIETY SERIES B, 1995. 57(1): p. 289.

Correspondence to:

Hui-Qi Qu, Ph.D.

Assistant Professor, University of Texas Health Science Center at Houston, School of Public Health, Brownsville Campus

80 Fort Brown, SPH Bldg., Brownsville, TX 78520

Ph 9568827006

Fax 9568825152

Email: Huiqi.Qu@uth.tmc.edu 